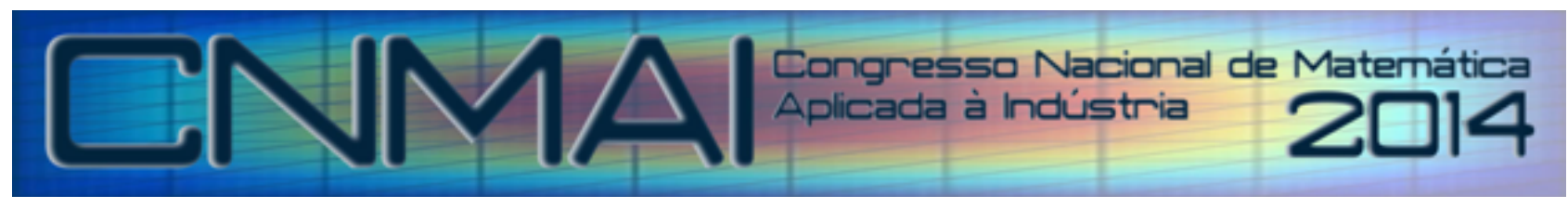

18 a 21 de novembro de 2014, Caldas Novas - Goiás

\title{
IDENTIFICAÇÃO DE UM LEVITADOR MAGNÉTICO NÃO LINEAR A PARTIR DE FUNÇÕES DE KAUTZ E ALGORITMOS GENÉTICOS
}

\author{
Elder Oroski, eoroski@gmail.com ${ }^{1}$ \\ João Roberto Deroco Martins, jrdmartins@yahoo.com.br ${ }^{2}$ \\ Adolfo Bauchspiess, adolfobs@ene.unb.br ${ }^{3}$ \\ ${ }^{1} \mathrm{UnB}$ - Departamento de Engenharia Elétrica \\ Campus Darcy Ribeiro \\ ${ }^{2}$ UNESP - Departamento de Engenharia Elétrica \\ Faculdade de Engenharia de Ilha Solteira \\ ${ }^{3} \mathrm{UnB}$ - Departamento de Engenharia Elétrica \\ Campus Darcy Ribeiro
}

\begin{abstract}
Resumo. Os problemas de identificação de sistemas tornam-se cada vez mais presentes nas áreas de Matemática aplicada e Engenharia de Controle. No que concerne à área industrial, muitos controladores de processos apresentam consideráveis melhorias de desempenho a partir de modelos do sistema a ser controlado. Neste contexto, este artigo aborda a identificação de um levitador magnético não-linear a partir de funções de base ortonormal, como as bases de Laguerre e de Kautz. Para a determinação dos coeficientes e dos pólos destas funções, é proposto um Algoritmo Genético, AG, auxiliado por operadores baseados nos métodos de Nelder Mead, NM, e Mínimos Quadrados, MMQ. Ao final do artigo, são apresentadas comparações entre os modelos obtidos a partir de diferentes números de funções na base ortonormal. Ainda, a efetividade do algoritmo proposto é mostrada pela sua comparação com um AG clássico.
\end{abstract}

Palavras-chave: Funções Ortonormais, Algoritmos Genéticos, Identificação de Sistemas

\section{INTRODUÇÃO}

Com inspiração nos conceitos de espaço vetorial da Álgebra Linear, as áreas de Controle de Sistemas vêm buscando uma base vetorial que pudesse explorar um espaço composto por funções de transferência. Entre as candidatas mais promissoras, encontram-se as funções de Laguerre, de Kautz e as GOBFs - Generalized Orthornormal Basis Functions, Heuberguer et al. (2005). Neste cenário, este artigo aborda a utilização de funções ortonormais discretas de Kautz, implementadas sob a forma de filtros digitais, para a identificação de um levitador magnético não linear. Para tanto, é apresentado um Algoritmo Genético, AG, cuja novidade é a utilização do algoritmo de Nelder Mead (NM) e o Método dos Mínimos Quadrados, MMQ, como otimizadores locais para obtenção dos parâmetros de modelo.

Para a determinação da estrutura do modelo, sabe-se, a priori, que as funções de Kautz se adaptam melhor à modelagem da dinâmica de sistemas subamortecidos, Machado et al. (2010). Como a resposta ao degrau do levitador magnético é pouco amortecida, tal função foi selecionada para construção da base vetorial que irá compor o modelo de sistema, a partir de suas combinações lineares e não lineares. A aplicação de funções ortonormais como base vetorial é interessante devido à ausência de realimentação de saída nas mesmas, fato desejável devido à não propagação de erros por recursão e principalmente por não ser necessário o conhecimento de termos passados do sinais de entrada e saída do sistema, Campello et al. (2007).

Para que sejam utilizadas as funcões ortonormais para composição de modelos de sistema, faz-se necessária a utilização de métodos de otimização para se encontrar os pólos de parametrização e os coeficientes destas 
funções. Métodos clássicos de otimização como o Gradiente Descendente e de Newton podem executar tal tarefa, porém necessitam de uma modelagem bem detalhada do sistema. Geralmente, é necessário o levantamento das derivadas bem como das matrizes Jacobianas e Hessianas do sistema, o que não se tem a priori, se o objetivo do processo for identificar o sistema.

Para contornar os problemas de otimização derminística geralmente são utilizados os métodos heurísticos, que podem obter resultados próximos do ótimo sem a necessidade de obtenção prévia das derivadas do sistema. Entre os métodos de otimização heurística mais conhecidos encontram-se o Particle Swarm Optimization, o Ant Colony Optimization e os Algoritmos Genéticos.

Na próxima seção será abordada a estrutura das funções ortonormais. Já na seção 3, são apresentados os principais aspectos relativos aos Algoritmos Genéticos e dos operadores de Nelder Mead e Mínimos Quadrados. Na seção 4 é detalhado o levitador magnético a ser identificado neste trabalho. Finalmente, na seção 5 são apresentados os resultados alcançados pelos Algoritmos Genéticos em comparação com os métodos determinísticos.

\section{FUNÇÕES ORTONORMAIS}

As funções ortonormais podem ser interpretadas como vetores, em espaços vetoriais de funções, que apresentam produto interno nulo entre si, Strang (2013). Tal condição é expresa em (1):

$$
\left\langle\psi_{i}, \psi_{j}\right\rangle= \begin{cases}0 & i \neq j \\ 1 & i=j\end{cases}
$$

sendo $\psi_{i}$ e $\psi_{j}$ funções ortonormais e $\langle$.$\rangle um produto interno adequado a tais funções contínuas, definido pela$ equação (2), segundo Heuberguer et al. (2005):

$$
\left\langle\psi_{i}(z), \psi_{j}(z)\right\rangle=\frac{1}{2 \pi i} \oint_{\mathcal{C}} \psi_{i}(z) \cdot \psi_{j}^{*}\left(1 / z^{*}\right) \frac{d z}{z},
$$

sendo $\mathcal{C}$ o círculo de raio unitário: $\mathcal{C}=\{z \in \mathbb{C}:|z \leq 1|\}$. Já paras as funções discretas, pode-se optar por um produto interno definido da seguinte forma:

$$
\left\langle\psi_{i}(z), \psi_{j}(z)\right\rangle=\sum_{k=0}^{\infty} \psi_{i}(k) \cdot \psi_{j}(k) .
$$

Assim, as funções ortonormais atenderão aos seguintes requisitos:

- $\psi_{j} \perp \psi_{j}$ para $i \neq j$, ou seja, as funções serão ortogonais entre si;

- $\left\|\psi_{n}\right\|=1, \forall n$, isto é, as funções apresentarão norma unitária.

A ortogonalidade é interessante para a base de funções, pois além das funções serem linearmente independentes, elas também possuem projeções nulas umas sobre as outras. Assim, tais funções são indicadas para compor a base vetorial $K$, base de Kautz, responsável por explorar o espaço vetorial composto por funções capazes de representar sistemas subamortecidos, Machado et al. (2010).

$$
K: \quad\left[\psi_{1}(z), \psi_{2}(z), \cdots, \psi_{n}(z)\right]
$$

Existe uma infinidade de funções ortonormais, de acordo com a definição de produto interno utilizada. As principais são: as funções de Hermit, Jacobi, Laguerre, Legendre, Kautz e as funções ortonormais generalizadas, em inglês: GOBF, Belt (1997).

\subsection{Funções de Laguerre}

As funções de Laguerre são funções ortonormais, parametrizadas por pólo real, Lemma et al. (2010), e podem ser expressas pela equação (5).

$$
L_{n}(z)=\frac{\sqrt{1-p^{2}}}{z-p}\left(\frac{1-p z}{z-p}\right)^{i-1}
$$

sendo $p$ o pólo das funções de Laguerre e $z$ a variável complexa associada a transformada $Z$.

Sendo $\mathbb{N}^{*}$ o conjunto dos inteiros não negativos, as funções de Laguerre podem compor uma base de funções ortonormais em $L_{2}\left(\mathbb{N}^{*}\right)$. Tal espaço vetorial é conhecido como espaço de Hilbert em $\mathbb{N}^{*}$ e é definido como o 
espaço das funções discretas, $\psi(k)$, quadraticamente somáveis, Belt (1997), ou seja, funções que atendem a equação (6).

$$
\sum_{n=1}^{\infty} \psi(k) \leq \infty
$$

A funções da base de Laguerre podem ser implementadas em forma de filtros digitais, sendo cada função associada a um filtro como exposto na figura 1.

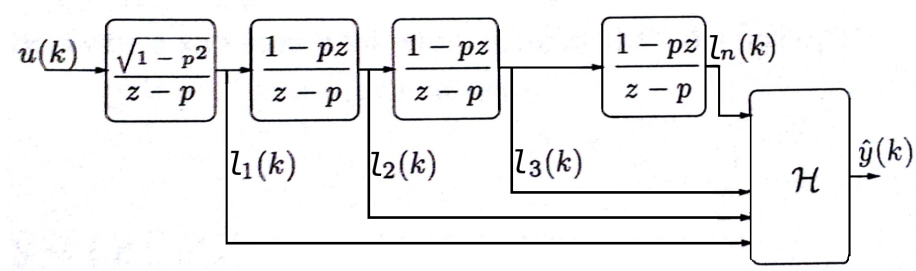

Figura 1. Modelo OBF com dinâmica de Laguerre.

No caso de sistemas Lineares e Invariantes no Tempo, LIT, o operador $\mathcal{H}$ pode ser definido como apenas a combinação linear das saídas dos filtros de Laguerre $l_{1}, l_{2}, \cdots, l_{n}$.

Já para os sistemas não lineares são necessárias combinações não lineares e combinações cruzadas entre as saídas dos filtros $l_{1}, l_{2}, \cdots, l_{n}$ para representar adequadamente tais sistemas.

\subsection{Funções de kautz}

As funções de Kautz são funções ortonormais, parametrizadas por pólo complexo, Lemma et al. (2010), e podem ser expressas pelas equações (7) e (8):

$$
\begin{gathered}
K_{2 m}(z)=\frac{\sqrt{\left(1-c^{2}\right)\left(1-b^{2}\right)}}{z^{2}+b(c-1) z-c} \cdot\left[\frac{-c z^{2}+b(c-1) z+1}{z^{2}+b(c-1) z-c}\right]^{m-1} \\
K_{2 m-1}(z)=\frac{z(z-b) \sqrt{1-c^{2}}}{z^{2}+b(c-1) z-c} \cdot\left[\frac{-c z^{2}+b(c-1) z+1}{z^{2}+b(c-1) z-c}\right]^{m-1}
\end{gathered}
$$

sendo $K_{2 m}(z)$ e $K_{2 m-1}(z)$ as funções pares e ímpares de Kautz, respectivamente. Já $\beta$ e $\bar{\beta}$ são os pólos, complexos conjugados, que parametrizam tais funções e os termos $b$ e $c$ podem ser expressos por (9) e (10):

$$
\begin{gathered}
b=(\beta+\bar{\beta}) /(1+\beta+\bar{\beta}), \\
c=-\beta \bar{\beta} .
\end{gathered}
$$

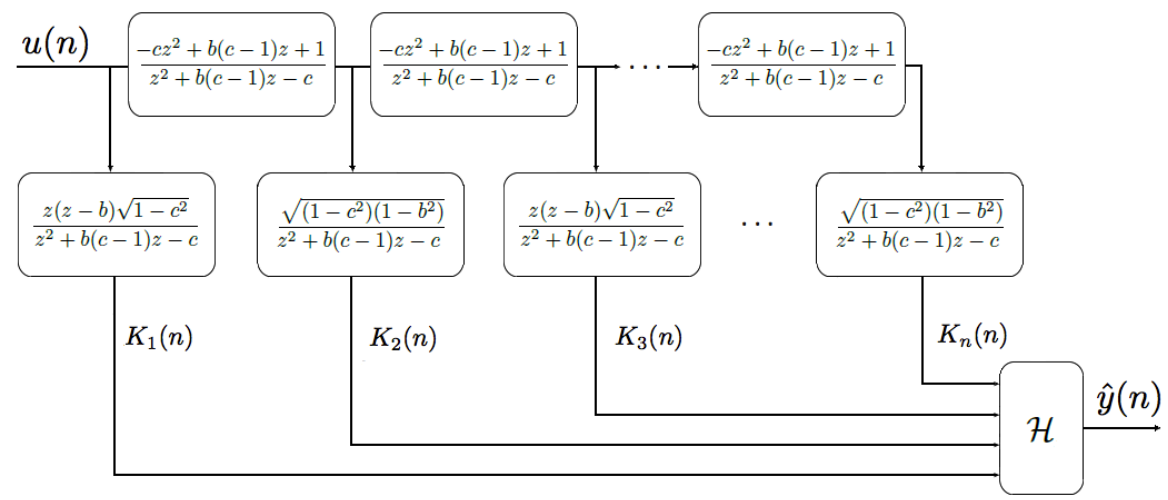

Figura 2. Modelo OBF com dinâmica de Kautz. 
O agrupamento de $n$ funções de Kautz pode formar uma base vetorial $K$, num espaço de funções $L_{2}\left(\mathbb{N}^{*}\right)$. Tal base é conhecida como base de Kautz e pode representar sistemas lineares da seguinte forma:

$$
\begin{array}{r}
M_{n k}(z)=c_{1} K_{1}(z)+c_{2} K_{2}(z)+\ldots+c_{N} K_{N}(z) \\
=\sum_{n=1}^{N} c_{n} K_{n}(z) .
\end{array}
$$

Já para os sistemas não lineares pode-se utilizar um modelo com a seguinte forma:

$$
\begin{array}{r}
M_{n k}(z)=c_{1} \cdot K_{1}(z)+c_{2} \cdot K_{2}(z)+\ldots+c_{N} \cdot K_{N}(z)+ \\
+c_{1,1} \cdot K_{1}(z) \cdot K_{1}(z)+c_{1,2} \cdot K_{1}(z) \cdot K_{2}(z)++c_{1,3} \cdot K_{1}(z) \cdot K_{3}(z) \ldots+c_{1, N} \cdot K_{1}(z) \cdot K_{N}(z)+ \\
+c_{2,2} \cdot K_{2}(z) \cdot K_{2}(z)+c_{2,3} \cdot K_{2}(z) \cdot K_{3}(z)+\ldots+c_{2, N} \cdot K_{2}(z) \cdot K_{N}(z)+ \\
+c_{3,3} \cdot K_{3}(z) \cdot K_{3}(z)+\ldots+c_{3, N} \cdot K_{3}(z) \cdot K_{N}(z)+ \\
\vdots \\
c_{n, n} \cdot K_{n}(z) \cdot K_{n}(z)= \\
N \sum_{n=1}^{N} c_{n} K_{n}(z)+\sum_{p=1}^{N} \sum_{q=p}^{N} c_{p, q} K_{p}(z) K_{q}(z) .
\end{array}
$$

Tal estrutura é uma variação das séries de Voltera, com truncamento no segundo núcleo, sendo as não linearidades tratadas nas saídas do modelo de forma semelhante à polinomial. Assim, tal modelo pode ser entendido como um modelo de Wiener, Aguirre (2007), conforme figura 3.

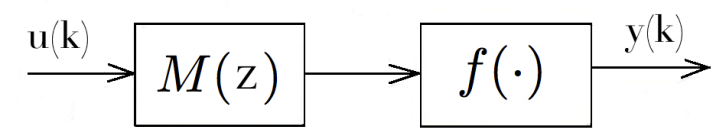

Figura 3. Modelo de Wiener, composto por um modelo dinâmico linear, $M(z)$, e uma não linearidade estática, geralmente polinomial, $f($.$) , Aguirre (2007).$

Os modelos de Winner são definidos como composição de um modelo dinâmico linear, $M(z)$, em cascata com uma função estática não linear, $f($.$) , Aguirre (2007).$

Para encontrar os pólos que parametrizam as funções de Kautz, $a+b i$, seus coeficientes, $c_{n}$, e seus graus de não linearidade, $g_{n}$, são utilizados Algoritmos Genéticos, AG, que serão apresentados na próxima seção.

\section{ALGORITMO GENÉTICO}

Os Algoritmos Genéticos são métodos heurísticos de otimização baseados nos princípios da evolução de Darwin, Koza (1994). Conforme o pseudocódigo, em um Algoritmo Genético clássico tem-se a criação aleatória de uma população de possíveis soluções ao problema tratado. Na sequência, tais soluções são avaliadas segundo sua adequação como solução de tal problema. Após tais passos, o algoritmo entra em um ciclo onde os melhores indivíduos, cromossomos, são probabilisticamente selecionados para serem progenitores da próxima geração. Os operadores de Cruzamento, Crossover e Mutação são aplicados à população e os novos indivíduos gerados são avaliados. Este ciclo repete-se até que o critério de parada seja alcançado.

Seguindo os princípios deste algoritmo espera-se que os indivíduos sofram um processo de seleção natural e os melhores indivíduos da população se aproximem da solução ótima do problema, Koza (1994).

\subsection{Parâmetros do Algoritmo Genético}

Em Algoritmos Genéticos, AG, cada indivíduo pertencente a população é definido por um cromossomo. Cada cromossomo é composto por genes que expressam os parâmetros a serem otimizados. Nesta formulação do AG, a codificação utilizada nos genes de um cromossomo é definida por:

$$
\begin{array}{|l|l|l|l|l|l|}
\hline c_{1} & c_{2} & \cdots & c_{n} & a & b \\
\hline
\end{array}
$$




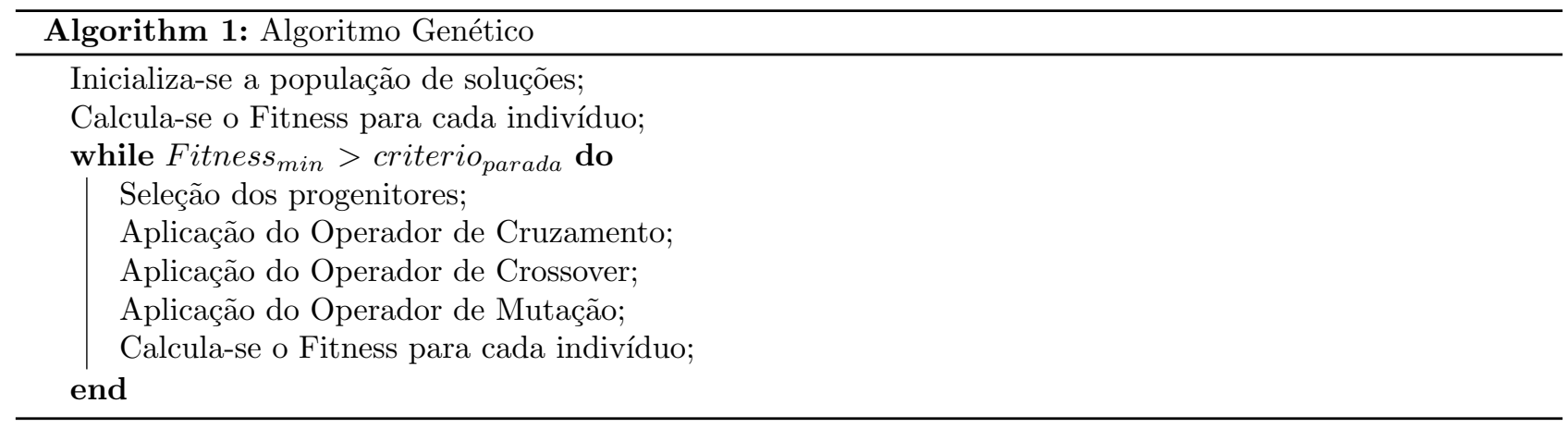

sendo $c_{1}, c_{2}, \cdots, c_{n}$ os coeficientes das funções ortonormais e $a+b i$ o pólo que as parametriza.

Os parâmetros do AG responsável pela otimização dos pólos e dos coeficientes das funções ortonormais são expressos na tabela 1 .

Tabela 1. Parâmetros do AG

\begin{tabular}{|l|c|}
\hline Parâmetro & Valor \\
\hline População & 200 Cromossomos \\
Met. de Seleção & Recombinação por Torneio \\
Taxa de Mutação & Variável $(20$ à $60 \%)$ \\
Taxa de Crossover & Variável $(80$ à $20 \%)$ \\
Nelder Mead & a cada 9 gerações \\
MMQ & a cada 7 gerações \\
\hline
\end{tabular}

Neste algoritmo o Fitness, métrica utilizada para medição do desempenho de um cromossomo, é baseado no MSE, Mean Square Error. As taxas de mutação e crossover são variáveis em relação ao número de gerações realizadas. A taxa de crossover se inicia em $80 \%$ buscando enfoque em buscas locais, sendo reduzida ao longo das gerações. Já a taxa mutação incia-se em 20\%, e é elevada com o passar das gerações, visando enfoque em buscas globais.

Cabe ressaltar que as taxas de mutação normalmente encontradas na literatura dificilmente ultrapassam 10\%. Neste trabalho tem-se taxas mais elevadas pela utilização de um operador de mutação baseado em perturbação do gene. Este baseia-se em somar uma constante aleatória, de pequeno módulo, ao gene em questão. Assim tem-se uma redução no efeito de busca aleatória normalmente gerada pelo operador de mutação, Koza (1994). No AG proposto neste artigo são utilizados dois operadores de busca local: o algoritmo de NM e o MMQ. Uma descrição completa do primeiro pode ser encontrada em Nelder (1965), enquanto o último é detalhado a seguir.

\subsection{Operador de Mínimos Quadrados}

O Método dos Mínimos Quadrados, MMQ, é uma das metodologias mais empregadas na área de Identificação de Sistemas. Assim, neste artigo propõem-se a utilização deste como um operador do Algoritmo Genético que "sintonizará" os parâmetros do modelo de funções ortonormais.

Este operador consiste na aplicação do MMQ, expresso pela equação (14), a um cromossomo escolhido aleatoriamente dentre a população de possíveis soluções.

$$
\hat{\theta}=\left(\Psi^{T} \Psi\right)^{-1} \Psi^{T} Y
$$

sendo $\theta$ o vetor de parâmetros do modelo e $\Psi$ a matriz de regressão, composta pelo sinal de entrada filtrado pelas funções ortonormais. Cabe ressaltar que, apesar do nome, a matriz $\Psi$ não possui versões regressivas do sinal de entrada, $u(n)$, e sim versões filtradas do mesmo. A matriz $\Psi$ possui suas colunas compostas pela equação (15).

$$
\check{K}_{n}(k)=\sum_{\tau=0}^{k} K_{n}(\tau) \cdot u(k-\tau)
$$

Se fosse utilizada a base de Kautz, os genes relativos ao pólo, em um cromossomo, seriam utilizados para gerar as funções de Kautz. Assim, a matriz $\Psi$ seria composta por versões filtradas, a partir de $K_{n}(k)$, do vetor de entrada $u(k)$. Tal estrutura é expressa na equação (16). 


$$
\Psi=\left[\begin{array}{cccc}
\vdots & \vdots & \vdots & \vdots \\
\check{K}_{1}(k) & \check{K}_{2}(k) & \cdots & \check{K}_{n}(k) \\
\vdots & \vdots & \vdots & \vdots
\end{array}\right]
$$

Após a ação do operador MMQ os genes relativos aos coeficientes das funções de Kautz são substituídos pelos elementos do vetor $\hat{\theta}$. Tal operador é utilizado no AG a cada ciclo de 7 gerações. Isto para evitar a convergência prematura das soluções a um mínimo local.

\section{LEVITADOR MAGNÉTICO}

O sistema escolhido neste artigo para aplicação das funções de Kautz é um levitador magnético, composto de duas bobinas magnéticas e um disco magnético móvel, cuja posição é função das correntes que percorrem as bobinas. Tal sistema é descrito na figura 4.

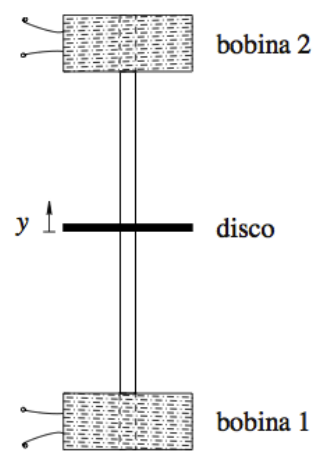

Figura 4. Levitador Magnético.

O sistema de levitação consiste em duas bobinas que produzem um campo magnético em resposta a uma excitação por corrente contínua. O disco movimenta-se por uma haste de vidro que minimiza o atrito, permitindo grandes deslocamentos verticais. Energizando-se a bobina inferior, um disco é levitado por uma força magnética repulsiva. Já para a bobina superior, a força de levitação é atrativa. Dois sensores, baseados em laser, medem as posições do disco, ECP (1999). Tal sistema pode ser descrito a partir das leis de Newton pela seguinte equação:

$$
m_{d} . \ddot{y}+c . \dot{y}=F_{b 1}-F_{b 2}-m_{d} . g
$$

sendo: $m_{d}$ a massa do disco, $c$ o coeficiente de atrito, $g$ a aceleração da gravidade e $F_{b 1}$ e $F_{b 2}$ as forças magnéticas exercidas pela bobina 1 e 2 respectivamente.ECP (1999). Tal sistema foi excitado a partir da seguinte forma de onda exposta na figura 5-a, enquanto que a saída obtida é mostrada na figura 5-b: 

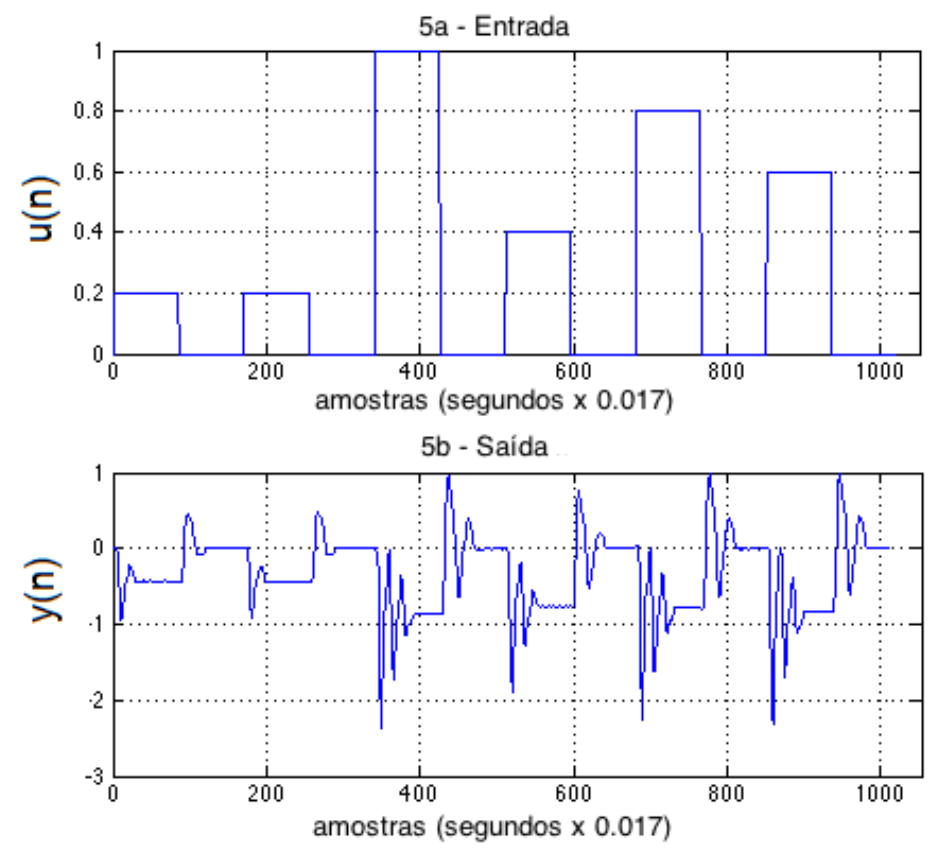

Figura 5. Levitador Magnético.

\section{RESULTADOS}

Os modelos foram obtidos a partir de vetores de entrada e saída de 1020 amostras do sistema. A validação foi realizada em vetores de dimensão 680. Os desempenhos dos modelos obtidos para o levitador magnético podem ser visualizados na tabela 2 .

Tabela 2. O erro médio quadrático, $M S E$, em relação ao $\mathrm{n}^{\mathrm{o}}$ de funções de Kautz que compõem o modelo.

\begin{tabular}{|c|c|c|c|}
\hline NF & pólo & $M S E_{\text {ident. }}$ & $M S E_{\text {valid. }}$ \\
\hline 2 & $\begin{array}{c}0.7782+ \\
0.3295 \mathrm{i}\end{array}$ & 0.0759 & 0.0886 \\
\hline 3 & $\begin{array}{c}0.7399+ \\
0.1836 \mathrm{i}\end{array}$ & 0.0434 & 0.0488 \\
& & \\
\hline 4 & $\begin{array}{c}0.8374+ \\
0.2670 \mathrm{i}\end{array}$ & 0.0212 & 0.0224 \\
\hline 5 & $\begin{array}{c}0.8552- \\
0.2639 \mathrm{i}\end{array}$ & 0.0147 & 0.0157 \\
& & 0.0091 \\
\hline 6 & $\begin{array}{c}0.7906+ \\
0.2800 \mathrm{i}\end{array}$ & 0.0085 & \\
\hline 7 & $0.8056+$ & 0.0074 & 0.0080 \\
& $0.2367 \mathrm{i}$ & & $\vdots$ \\
\hline$\vdots$ & $\vdots$ & $\vdots$ & 0.0023 \\
\hline 20 & $0.7906+$ & $6.545 .10^{-4}$ & \\
& $0.2800 \mathrm{i}$ & & \multicolumn{2}{|c}{} \\
\hline
\end{tabular}

sendo: $M S E_{\text {indent. e }} M S E_{\text {valid. }}$ os erros médios quadráticos na etapas de identificação e validação, respectivamente. Estes resultados foram obtidos após 200 gerações do AG, com auxílio dos operadores NM e MMQ.

O modelo apontado na linha 1 da tabela 2 pode ser visualizado na figura 6 . Este modelo, utilizando 2 funções de Kautz, é expresso na equação (18):

$$
\begin{array}{r}
M_{2 K}(z)=-6.8077 . \check{K}_{1}(z)+3.5903 \cdot \check{K}_{2}(z)+2.9948 .\left[\check{K}_{1}(z)\right]^{2}- \\
-2.1374 \cdot \check{K}_{1}(z) \cdot \check{K}_{2}(z)+2.3279 \cdot\left[\check{K}_{2}(z)\right]^{2} .
\end{array}
$$

O modelo apontado na linha 5 da tabela 2, utilizando 6 funções de Kautz, pode ser visualizado na figura 7 . Este modelo, composto por 6 funções de Kautz, é expresso pela equação (19). 


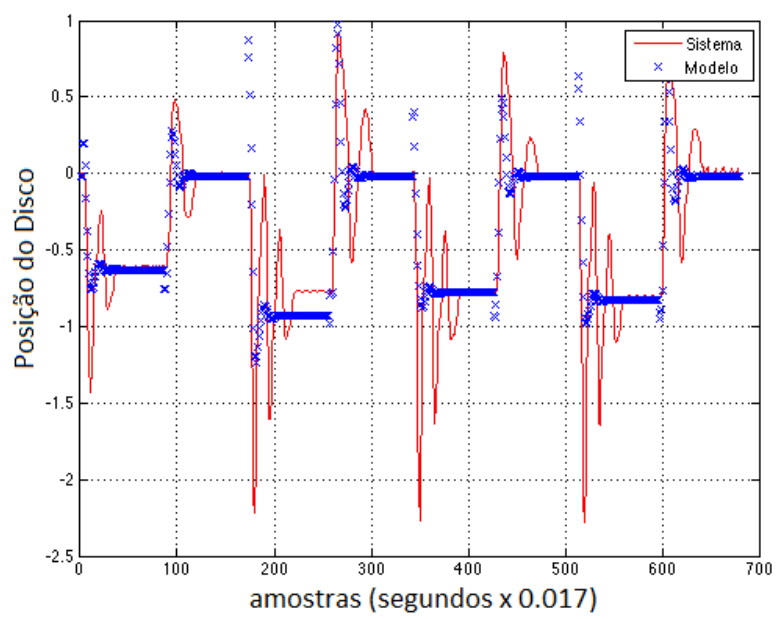

Figura 6. Modelo para o Levitador, a partir da base de Kautz, utilizando 2 funções.

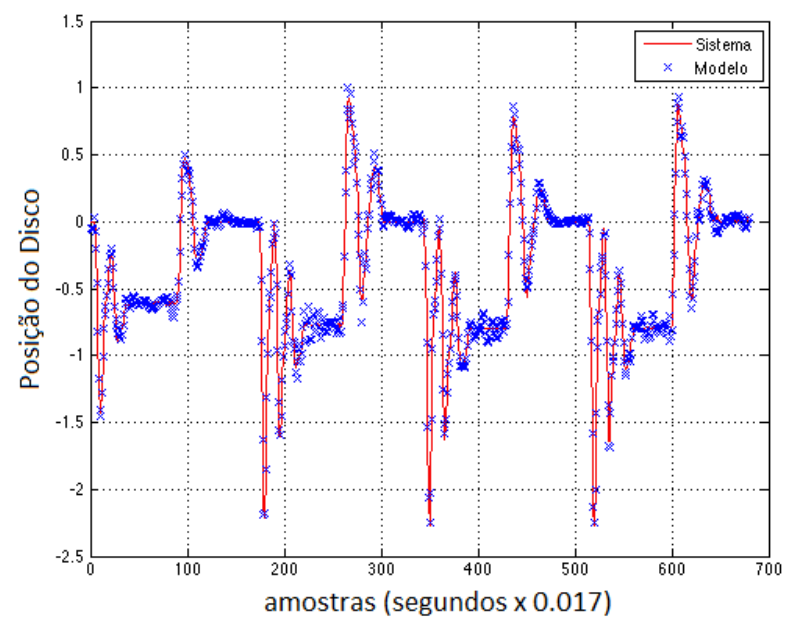

Figura 7. Modelo para o Levitador, a partir da base de Kautz, utilizando 6 funções.

$$
M_{6 K}(z)=\sum_{n=1}^{6} a_{n} \cdot \check{K}_{n}(k)+\sum_{p=1}^{6} \sum_{q=p}^{6} b_{p, q} \cdot \check{K}_{p}(k) \cdot \check{K}_{q}(k)
$$

sendo $a_{n}$ e $b_{p, q}$ expressos nas tabelas 3 e 4 :

Tabela 3. Parâmetro $a_{n}$ do modelo (19).

\begin{tabular}{|c|cccccc|}
\hline $\mathrm{n}$ & 1 & 2 & 3 & 4 & 5 & 6 \\
\hline$a_{n}$ & -6.0409 & 2.7393 & -0.8848 & -4.9437 & 2.2708 & -2.5762 \\
\hline
\end{tabular}

Tabela 4. Parâmetro $b_{p, q}$ do modelo (19)

\begin{tabular}{|c|cccccc|}
\hline$b_{p, q}$ & 1 & 2 & 3 & 4 & 5 & 6 \\
\hline 1 & -1.5398 & -6.9036 & 8.3240 & 0.9579 & -5.2464 & 5.5990 \\
2 & - & 1.3529 & 15.7666 & -1.2997 & -11.3885 & 1.8267 \\
3 & - & - & 0.4880 & -2.5214 & -1.7015 & -5.5351 \\
4 & - & - & - & 1.7455 & 7.1258 & -0.5095 \\
5 & - & - & - & - & 1.1335 & -0.3490 \\
6 & - & - & - & - & - & 2.1309 \\
\hline
\end{tabular}


A convergência do AG, empregado na obtenção do modelo expresso na linha 3 da tabela 2 pode ser observada na figura 8, com e sem os operadores NM e MMQ:

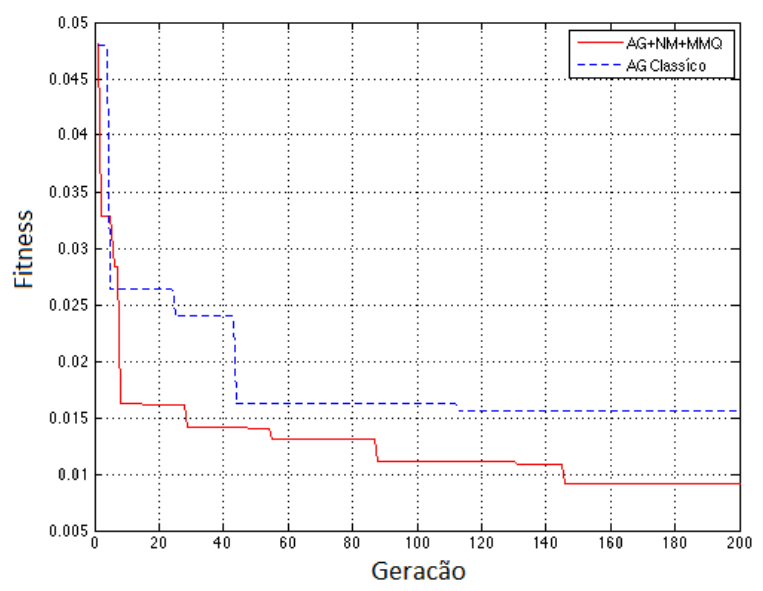

Figura 8. Convergência do Algoritmo Genético clássico (linhas tracejadas) e com os operadores NM e MMQ (linhas contínuas).

Para efeitos de Benchmark, o mesmo processo de identificação foi tratado por Da Rosa (2009) a partir do método de Levenberg Marquardt. Os resultados por ele obtidos são expostos na figura 9.

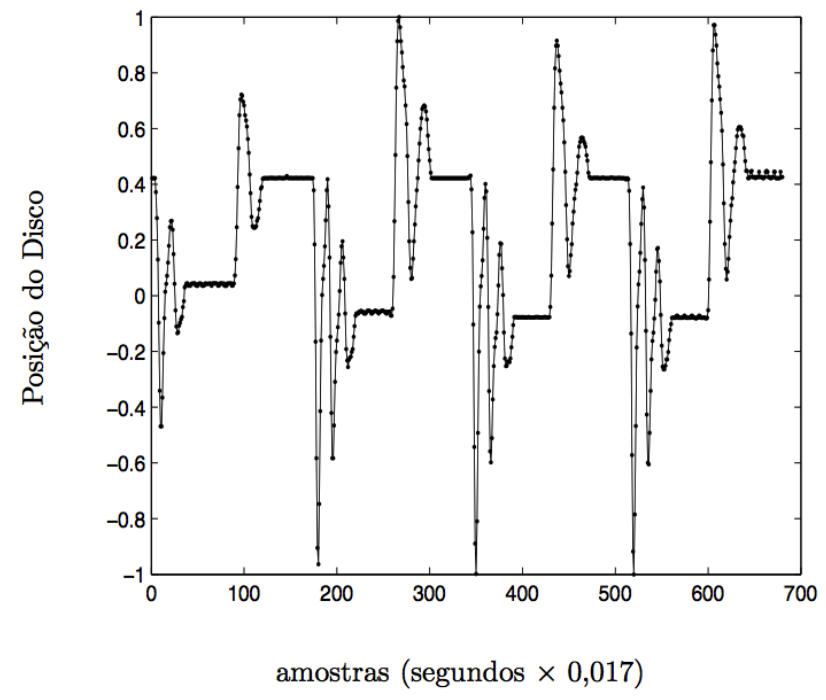

Figura 9. Resultados obtidos por Da Rosa (2009) na identificação do levitador magnético.

Pode-se perceber que os resultados de Da Rosa (2009) são sensivelmente mais exatos que os resultados obtidos pelo Algorimo Genético proposto. Entretanto, o método exposto por Da Rosa necessita do cálculo de gradientes envolvidos no processo de otimização. Já a metodologia proposta, independe de tais equacionamentos e ainda assim obtém resultados próximos do ótimo.

\section{CONCLUSÃO}

Neste artigo foi discutida a modelagem de sistemas não lineares a partir de funções de base ortonormal de Kautz em estrutura de série de Voltera. Para determinação de seus parâmetros, foi utilizado um AG acelerados pelos operadores de NM e MMQ.

Pode-se concluir que a implementação do AG que contava com os operadores de Nelder Mead e MMQ convergiu mais rapidamente que a implementação de AG clássico. Nas comparações dos resultados obtidos com os resultados de Da Rosa (2009) pode-se perceber que os resultados deste são sensivelmente mais acurados, mas dependem do equacionamento dos gradientes envolvidos no problema de otimização. Já nos resultados expostos neste artigo, tem-se um resultado muito próximo do obtido por Da Rosa, com a vantagem da não necessidade dos gradientes.

Vislumbra-se, como trabalhos futuros, a possibilidade de aplicação de Programação Genética (PG) para a determinação do grau de não linearidade do sistema, bem como dos parâmetros do modelo não linear. 


\title{
7. AGRADECIMENTOS
}

Ficam os agradecimentos ao Prof Alex da Rosa, da UnB, que nos instruiu sobre a manipulação dos dados de entrada e saída utilizados neste trabalho.

\section{REFERÊNCIAS}

AGUIRRE, Luis Antônio. 2007. Introdução a Identificação de Sistemas - Técnicas Lineares e não lineares Aplicadas a Sistemas Reais. Editora UFMG, $3^{a}$ Ed.

BELT WILLEM , H. 1997. Orthonormal Bases for Adaptative Filtering. Phd thesis, Technische Universiteit Eindhoven.

CAMPELlO, R., AMARAL, W. C., e OLIVEIRA G. 2007. Identificação de Controle de Processsos Via Desenvolvimento de Séries Ortonormais. Revista Controle e Automação, vol. 18, (pp 301-319).

ECP. 1999. Manual for Model 730, Magnetic Levitation System, Educational Control Products. California, USA.

DA ROSA, Alex, CAMPELlO, R., AMARAL, W. C. 2009. Exact Search Directions for Optimization of Linear and Nonlinear Models Based on Generalized Orthonormal Functions. IEEE Transactions on Automatic Control, vol. 54, $N^{o}$ 12, (pp 2757-2771).

HEUBERGER, P., VAN DEN HOF, P. and WAHLBERG, B. 2005. Modelling and Identification with Rational Orthogonal Basis Functions. Springer Press, $1^{\text {st }}$ Ed.

KOZA, John. 1994. Genetic Programming: On the Programming of Computers by Means of Natural Selection. MIT Press, $6^{\text {th }}$ Ed.

LEMMA, D., RAMASSAMY, M. and SHUHAIMI, G. M.. 2010. System Identification Using Orthonormal Basis Filter. Journal of Applied Sciences, vol. 10, (pp 2516-2522).

MACHADO, J., CAMPELLO, R., e AMARAL, W. 2010. Modelos Lineares GOBF com Funções Internas. VIII Congresso Brasileiro de Automática, (pp. 505-512).

NELDER, J. A. and MEAD, R. 1965. A simplex for function minimization. Computer Journal, Vol. 7, (pp 308-313).

STRANG, Gilbert. 2013. Álgebra Linear e suas Aplicações. Cengage Learning, $4^{a}$ Ed.

\section{RESPONSABILIDADE AUTORAL}

"Os autores são os únicos responsáveis pelo conteúdo deste trabalho".

\section{Identification of a Magnetic Levitator using Kautz Functions and Genetic Algorithm}

\author{
Elder Oroski, eoroski@gmail.com ${ }^{1}$ \\ João Roberto Deroco Martins, jrdmartins@yahoo.com.br ${ }^{2}$ \\ Adolfo Bauchspiess, adolfobs@ene.unb.br ${ }^{3}$ \\ ${ }^{3} \mathrm{UnB}$ - Departamento de Engenharia Elétrica \\ Campus Darcy Ribeiro \\ ${ }^{4}$ UNESP - Departamento de Engenharia Elétrica \\ Faculdade de Engenharia de Ilha Solteira \\ ${ }^{5}$ UnB - Departamento de Engenharia Elétrica \\ Campus Darcy Ribeiro
}

\begin{abstract}
The presence of problems concerning systems identification, in areas such as applied Mathematics and Control engineering, is increasingly growing. Many controllers, used in industrial processes, have had its performance improved using models that describe the system to be controlled. In this context, this paper discusses the identification of a nonlinear magnetic levitator, using orthonormal basis functions, such as Laguerre and Kautz basis. In order to determine the coefficients and the poles of these functions, a Genetic Algorithm, GA, is proposed. This algorithm is aided by operators based in the Nelder Mead, NM, and Least Mean Squares, LMS, methods. Models were obtained using different numbers of functions in the orthonormal basis. The results were, then, compared. The effectiveness of the algorithm proposed is also shown by comparison with a classic GA.
\end{abstract}

Keywords: System Identification, Orthonormal Functions, Genetic Algorithm 